\title{
MR Spectroscopy Shows Long Propylene Glycol Half-Life in Neonatal Brain
}

\author{
Monique van de Lagemaat ${ }^{a}$ Laura A. van de Pol ${ }^{b}$ Inge A. Zonnenberg ${ }^{a, c}$ \\ Bregje C.M. Witjes ${ }^{d}$ Petra J.W. Pouwels ${ }^{\mathrm{e}}$ \\ aDepartment of Neonatology, Emma Children's Hospital Amsterdam UMC, Amsterdam, The Netherlands; \\ bepartment of Pediatric Neurology, Emma Children's Hospital Amsterdam UMC, Amsterdam, The Netherlands; \\ 'Department of Neonatology, Wilhelmina Children's Hospital University Medical Center Utrecht, Utrecht, The \\ Netherlands; 'Department of Pharmacy, Erasmus MC Sophia Children's Hospital, Rotterdam, The Netherlands; \\ eDepartment of Radiology and Nuclear Medicine, Amsterdam UMC, Amsterdam, The Netherlands
}

\section{Keywords}

Neonate $\cdot$ Propylene glycol · MR spectroscopy $\cdot$ Half-life

\begin{abstract}
Introduction: Neonatal propylene glycol (PG) clearance is low with long plasma half-life. We hypothesized that neonatal brain PG clearance is diminished and may be related to perinatal asphyxia, infection, or stroke, via different bloodbrain barrier permeability. This study aimed to estimate cerebral PG half-life with a clearance model including PG measured with MR spectroscopy (MRS) in neonates that received phenobarbital as the only PG source and to evaluate whether PG clearance was related to intracerebral pathology, for example, perinatal asphyxia, infection, or stroke. Methods: In this retrospective cohort study, 45 neonates receiving any dose of phenobarbital underwent MRS (short echo time single-voxel MRS at 1.5 T). Cumulative phenobarbital/PG doses were calculated. MRS indications were perinatal asphyxia $(n=22)$, infection $(n=4)$, stroke $(n=10)$, metabolic disease $(n=4)$, and others $(n=5)$. Results: Medians (interquartile range) included gestational age 39.4 (3.1) weeks, birth
\end{abstract}

karger@karger.com www.karger.com/neo

Karger

GOPEN ACCESS
(C) 2021 The Author(s)

Published by S. Karger AG, Basel

This is an Open Access article licensed under the Creative Commons Attribution-NonCommercial-4.0 International License (CC BY-NC) (http://www.karger.com/Services/OpenAccessLicense), applicable to the online version of the article only. Usage and distribution for commercial purposes requires written permission. weight $3,146(1,340) \mathrm{g}$, and cumulative PG dose $700(1,120)$ $\mathrm{mg} / \mathrm{kg}$. First-order kinetics with mono-exponential decay showed cerebral PG half-life of $40.7 \mathrm{~h}$ and volume of distribution of $1.6 \mathrm{~L} / \mathrm{kg}$. Zero-order kinetics showed a rate constant of $0.048 \mathrm{~mm} / \mathrm{h}$ and a volume of distribution of $2.3 \mathrm{~L} / \mathrm{kg}$, but the fit had larger residuals than the first-order model. There were no differences in $\triangle P G$ (i.e., $P G$ estimated with clearance model minus PG observed with MRS) in infants with perinatal asphyxia, infection, or stroke. Discussion/Conclusion: This study showed a long cerebral PG half-life of $40.7 \mathrm{~h}$ in neonates, unrelated to perinatal asphyxia, infection, or stroke. These findings should increase awareness of possible toxic $P G$ concentrations in neonatal brain due to intravenous PG-containing drugs.

(c) 2021 The Author(s)

Published by S. Karger AG, Basel

\section{Introduction}

Propylene glycol (PG) improves drug solubility and stability, also for intravenous phenobarbital used neonatally. Hepatic alcohol dehydrogenase metabolizes PG to 
lactate and pyruvate; PG is eliminated renally $[1,2]$ (Fig. 1). PG clearance is saturable with non-linear pharmacokinetics after adult high doses [1]; however, lower neonatal concentrations show first-order kinetics with dose-dependent elimination [3]. Neonates have low PG clearance due to immature hepatic and renal function compared to adults [3-5]. In neonates, plasma PG halflife varies between 10.8 and $30.5 \mathrm{~h}[3,6]$. Consequently, neonates are at risk of PG accumulation and toxicity. Furthermore, with fast passive diffusion via the blood-brain barrier (BBB), long neonatal PG plasma half-life promotes cerebral accumulation and potential toxicity.

PG is generally regarded as safe [7]; the neonatal safety threshold is $1 \mathrm{mg} / \mathrm{kg} /$ day [8]. Daily PG doses exceeding safety thresholds are regularly administered to hospitalized neonates via PG-containing drugs $[4,5,9,10]$. We demonstrated previously that administration of different pharmaceutical formulations with cumulative phenobarbital doses up to $40 \mathrm{mg} / \mathrm{kg}$ results in median cumulative PG doses up to $1,400 \mathrm{mg} / \mathrm{kg}$ [10]. This exceeds recommended safety thresholds $[7,8]$ and doses for which no short-term adverse effects were observed [9]. Short-term adverse PG effects include lactic acidosis, convulsions, haemolysis, and renal, hepatic, cardiac, or respiratory failure $[6,8,11]$; long-term adverse effects are largely unknown. Furthermore, high cumulative PG doses cause high brain PG concentrations on MR spectroscopy (MRS), affected by dose-to-MRS interval [10].

The BBB encompasses the microvascular endothelium with highly restrictive tight junctions between blood, cerebrospinal fluid (CSF), and the brain [12-14]. Neonatal $\mathrm{BBB}$ disruption can be caused by various pathological conditions, including perinatal asphyxia $[12,14]$, infection [13], and stroke [15], possibly leading to higher cerebral concentrations of potentially toxic molecules [14].

In this study, we estimated cerebral PG half-life based on MRS-detected PG in neonates that received phenobarbital, as the only PG source. We evaluated whether cerebral PG half-life was related to underlying diagnosis with possibly different BBB permeability.

\section{Materials and Methods}

\section{Subjects}

This retrospective cohort study included neonates born at 2442 weeks gestational age in our neonatal intensive care unit between January 2016 and January 2019, as described previously [10], but amended with prolonged inclusion. In short, neonates were included if cerebral MRI/MRS at $1.5 \mathrm{~T}$ was performed between birth and 4 weeks after term equivalent age. Seventy-two infants were eligible for inclusion. Six infants with MRI at $3 \mathrm{~T}$ and 3 with insufficient MRS quality (movement artefacts) were excluded. Consequently, the original cohort included 63 neonates. The present study included 45 neonates receiving any dose of phenobarbital (sole PG source). The online supplementary material (see www.karger.com/doi/10.1159/000519282 for all online suppl. material) includes a STROBE checklist.

MRI/MRS indications were perinatal asphyxia $(n=22)$; infection $(n=4)$; stroke $(n=10)$; metabolic disease $(n=4)$, including hypoglycaemia $(n=3)$ and hyperammonaemia $(n=1)$; and other diagnoses $(n=5)$, including SCN2A mutation epilepsy $(n=2)$, hemimegalencephaly $(n=1)$, intoxication $(n=1)$, and 5th-day-fits $(n=1)$. Perinatal asphyxia was defined as $\geq 1$ of the following: Apgar score $\leq 5$ at 5 min; cardiopulmonary resuscitation; postnatal mechanical ventilation $>10 \mathrm{~min}$; or $\mathrm{pH}<7.10$, base excess $<-16$ $\mathrm{mmol} / \mathrm{L}$, or lactate $>10 \mathrm{mmol} / \mathrm{L}$ in the first postnatal hour. Perinatal infection included proven sepsis (i.e., positive blood culture) or clinical sepsis (i.e., clinical symptoms, elevated infection parameters, antibiotics, and negative blood culture) [16]. Perinatal stroke was MRI-diagnosed. Metabolic disease was diagnosed with specific biochemical characteristics.

Birth and phenobarbital dosing data were retrospectively collected from medical records. Birth data included gestational age (weeks), birth weight (g), mortality, and sex. Phenobarbital dosing data included time of administration, dosage $(\mathrm{mg} / \mathrm{kg})$, and injection solution, that is, $50 \mathrm{mg} / \mathrm{mL}$ (TEVA Pharmachemie, Haarlem, the Netherlands), $25 \mathrm{mg} / \mathrm{mL}$ (Apotheek A15, Gorinchem, the Netherlands), or $10 \mathrm{mg} / \mathrm{mL}$ phenobarbital (hospital pharmacy of Haarlem and Eindhoven, the Netherlands). All solutions contained $350 \mathrm{mg} / \mathrm{mL}$ PG and 336, 240, 329, and $237 \mathrm{mg} / \mathrm{mL}$ ethanol, respectively. Based on the phenobarbital dosages, the cumulative phenobarbital and PG dose ( $\mathrm{mg} / \mathrm{kg}$ ) administered before MRS was calculated. Phenobarbital dose-to-MRS intervals (h) and postnatal age at MRS (h) were determined.

\section{MRS Acquisition and Analysis}

During MRI, neonates were placed on a special cushion for adequate positioning and preventing movements. Most infants were mechanically ventilated and intravenously received pain relief with morphine and/or sedation with midazolam, without PG, ethanol, or other alcohol-based excipients. In some infants, midazolam was anticonvulsive. Infants without mechanical ventilation were fed prior to MRI and not sedated.

Clinical conventional MRI was performed at $1.5 \mathrm{~T}$ (Avanto, Siemens, Erlangen, Germany) using an 8-channel head coil [10]. ${ }^{1} \mathrm{H}$-MRS was performed with point-resolved spectroscopy localization at the right basal ganglia and thalamus with $14 \mathrm{~mL}$ volume of interest (VOI; $20 \times 35 \times 20 \mathrm{~mm}$ RL-AP-SI) [10]. Five subjects had slightly smaller VOI $(8-12 \mathrm{~mL}) ; 4$ subjects had VOI accidentally selected in the parietal cortex ( $8 \mathrm{~mL}[n=3], 27 \mathrm{~mL}[n=1])$ - these spectra were included. The repetition time was 3,000 ms, echo time $30 \mathrm{~ms}$, and 32 averages (64 averages in 4 subjects). Metabolite concentrations in millimolar (i.e., $\mathrm{mmol} / \mathrm{L} \mathrm{VOI}$ ) were calculated with LCModel [17], using a standard basis set including lactate and additional model spectra of PG [18], pyruvate, ethanol, and simulating macromolecular and lipid signals. Quality parameters included spectral linewidth (full-width-at-halfmaximum $[\mathrm{Hz}]$ ) and signal-to-noise ratio (SNR), as normalized SNR corresponding to the standard protocol of VOI $14 \mathrm{~mL}$ and 32 averages. 
Fig. 1. Propylene glycol metabolism in humans. $\mathrm{ADH}$, alcohol dehydrogenase; ALDH, aldehyde dehydrogenase.

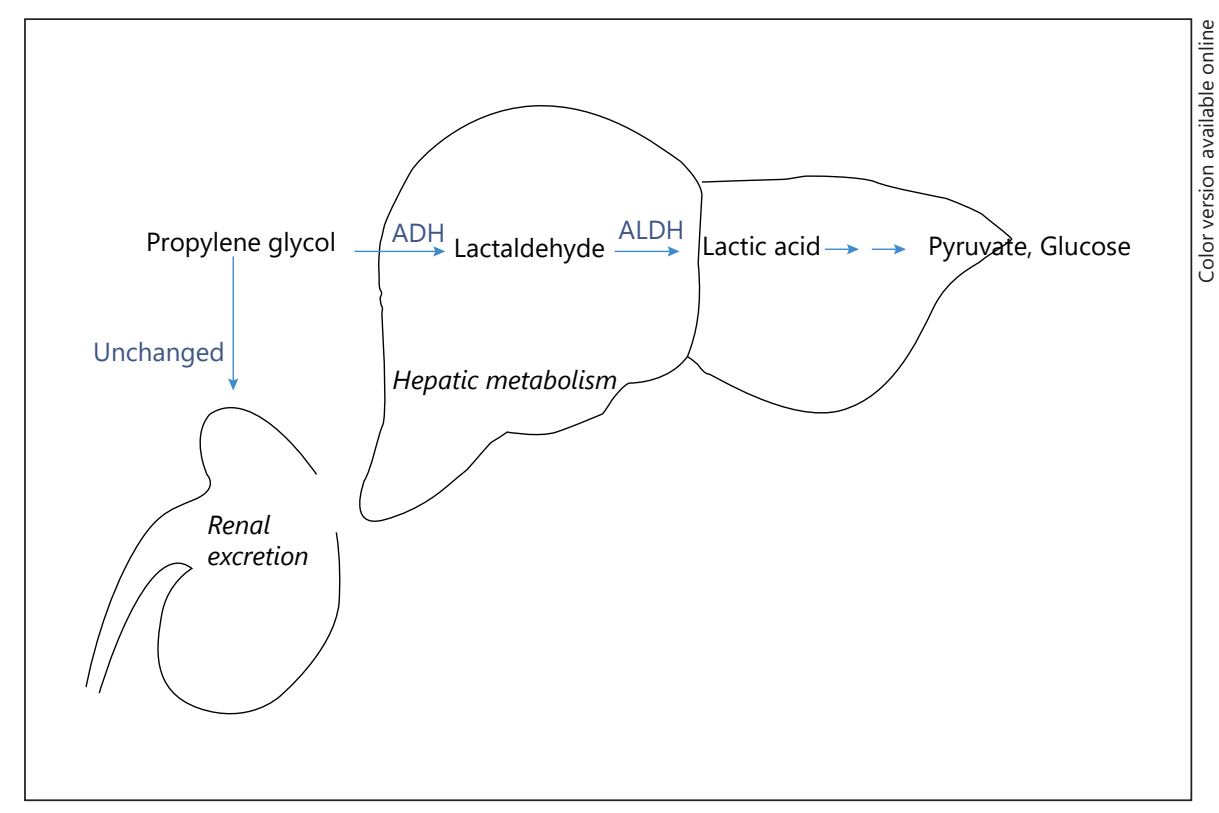

\section{Cerebral PG Clearance Model}

We expressed estimated cerebral PG concentration as a function of PG dose and dose-to-MRS interval, assuming the same values for half-life and volume of distribution for all subjects. Based on PG dosages $P G_{\mathrm{i}}(\mathrm{mmol} / \mathrm{kg})$ and administration-to-MRS interval $\Delta t_{\mathrm{i}}$, we assumed first-order kinetics:

$$
P G_{\text {estimated }}=\sum_{i} P G_{i} / V_{d} \times \exp \left(-\Delta t i / t_{1 / 2}\right) .
$$

First-order model: PG half-life $t_{1 / 2}(\mathrm{~h})$ and volume of distribution $V d$ (L/kg brain tissue) were estimated by means of least squares optimization between $P G_{\text {estimated }}$ and $P G_{\text {observed }}$ (i.e., measured with MRS). Based on this clearance model, $P G_{\text {predicted }}$ was calculated per participant.

Zero-order kinetics is described in adults [1] and suggested for high neonatal PG dosages $[3,19]$. Therefore, also a zero-order kinetics model was analysed, assuming the same values for the zeroorder rate constant $k$ and volume of distribution $V d$ for all subjects:

Zero-order model:

$$
P G_{\text {estimated }}=\sum_{i}\left(P G_{i} / V_{d}-k \Delta t_{i, i+1}\right) .
$$

$\Delta t_{i, i+1}$ : interval between subsequent phenobarbital/PG administrations (in case of multiple doses), and for the final dose the dose-to-MRS interval. In this equation, $P G_{\text {estimated }}$ cannot become negative. Parameters $k$ and $V d$ were estimated by means of least squares optimization between $P G_{\text {estimated }}$ and $P G_{\text {observed }}$. To estimate whether $P G$ kinetics is influenced by diagnosis, we compared $\triangle \mathrm{PG}$ (differences between $P G_{\text {predicted }}$ [i.e., with PG clearance model] and $P G_{\text {observed }}$ [i.e., with MRS]) between diagnoses.

\section{Statistics}

Categorical variables were reported as frequencies (\%) and analysed using $\chi^{2}$ tests. Continuous variables were skewed and re-
Table 1. Demographics and clinical data of the study population

\begin{tabular}{ll}
\hline Population characteristics & $n=45$ \\
\hline Male, $n$ (\%) & $26(58)$ \\
Mortality, $n$ (\%) & $13(29)$ \\
$\quad$ Postnatal age at death, h & $182(166)$ \\
Gestational age, weeks & $39.4(3.1)$ \\
Birth weight, g & $3,146(853)$ \\
Phenobarbital administration ${ }^{\mathrm{a}}$ & \\
$\quad$ Phenobarbital total dose, mg/kg & $40(20)$ \\
PG total dose, mg/kg & $700(1,120)$ \\
$\quad$ Interval last dose phenobarbital to MRI, h & $29.2(80.7)$ \\
Cerebral MRS & \\
$\quad$ Postnatal age at MRS, h & $104(64)$ \\
Postmenstrual age at MRS, weeks & $40.4(3.1)$ \\
SNR & $15.9 \pm 4.2$ \\
FWHM, Hz & $1.5(0.51)$ \\
MRS PG, mM & $1.7(3.9)$ \\
MRS lactate, mM & $0.8(0.7)$ \\
\hline
\end{tabular}

Parameters are reported as frequencies (\%), median (IQR), or mean \pm SD. FWHM, full-width at half-maximum; MRS, MR spectroscopy; PG, propylene glycol; SNR, signal-to-noise ratio; SD, standard deviation; $\mathrm{IQR}$, interquartile range. ${ }^{\text {a }}$ Phenobarbital was the only medication containing PG (all formulations contained $350 \mathrm{mg} / \mathrm{mL}$ PG).

ported as median (interquartile range) and analysed using MannWhitney-U and Kruskal-Wallis tests. Normally distributed SNR was reported as mean \pm SD and analysed using ANOVA. All analyses included post hoc Bonferroni correction for multiple testing. As lactate is a PG metabolite (Fig. 1), it was evaluated whether lactate and PG measured with MRS were correlated using Spearman's rho. All analyses were performed using IBM SPSS Statistics version 26. A $p$ value $<0.05$ was considered significant. 

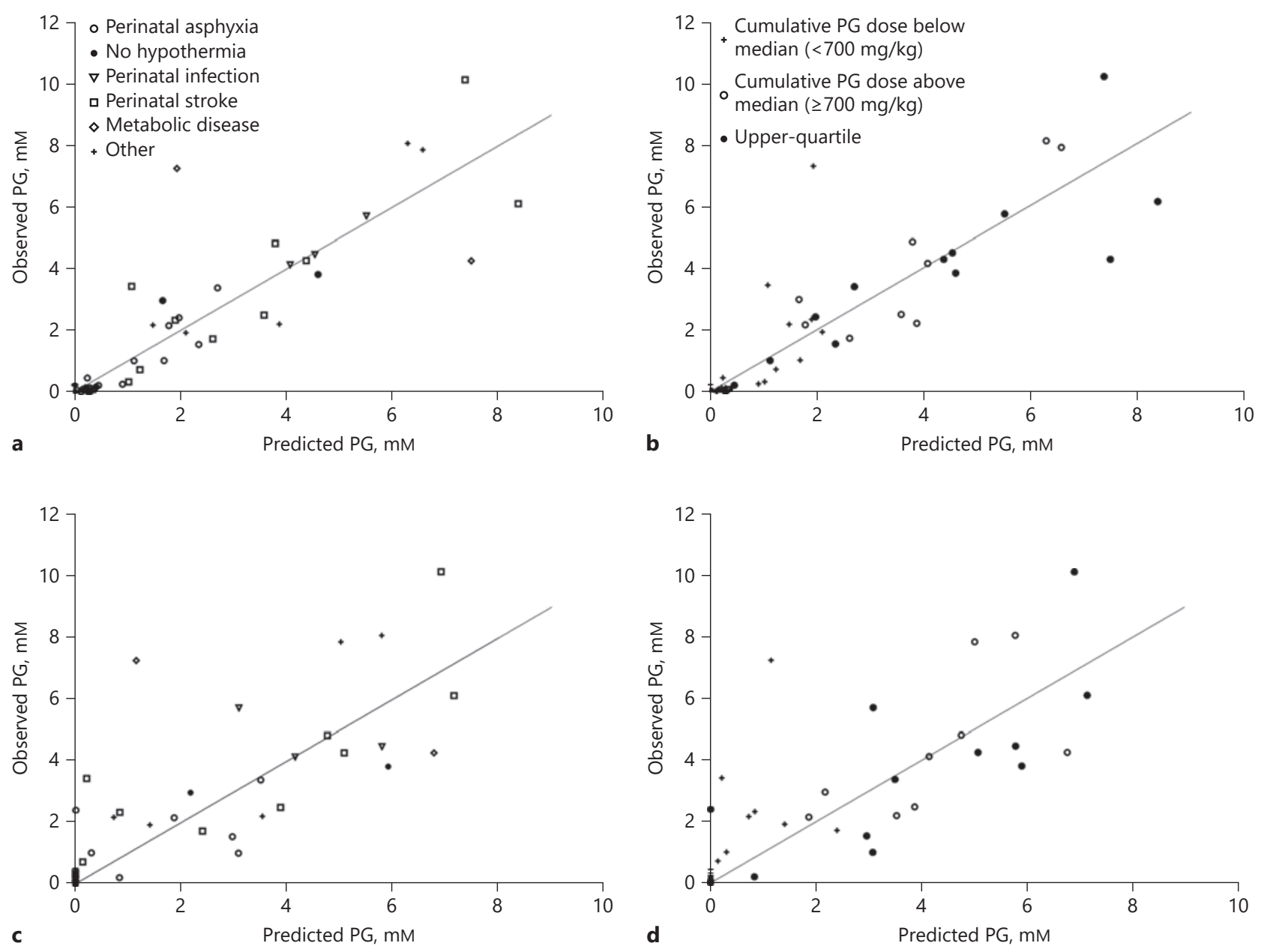

Fig. 2. $P G$ clearance model. First-order model: $P G_{\text {observed }}$ measured with MRS versus $P G_{\text {predicted }}$ based on least-squares optimization estimated a PG half-life of $40.7 \mathrm{~h}$ and a volume of distribution of $1.6 \mathrm{~L} / \mathrm{kg}(n=45)$ classified by diagnosis (a); classified by cumulative PG dose above and below the median (i.e., $700 \mathrm{mg} / \mathrm{kg}$ ) (b). Zero-order model: $P G_{\text {observed }}$ measured with MRS versus

$P G_{\text {predicted }}$ based on least-squares optimization estimated a PG rate constant of elimination of $0.048 \mathrm{~mm} / \mathrm{h}$ and a volume of distribution of $2.35 \mathrm{~L} / \mathrm{kg}(n=45)$ classified by diagnosis (c); classified by cumulative PG dose above and below the median (i.e., $700 \mathrm{mg} / \mathrm{kg}$ ) (d). Line of identity was plotted in (a-d). MRS, MR spectroscopy; PG, propylene glycol.

\section{Results}

\section{Subjects}

The gestational age was 39.4 (3.1) weeks, birth weight 3,146 (853) g, and chronological age at MRI/MRS 104 (64) h (Table 1). After the first phenobarbital dose (20 $\mathrm{mg} / \mathrm{kg}$ according to national guidelines), 33 neonates received a second $(10 \mathrm{mg} / \mathrm{kg}), 21 \mathrm{a}$ third $(10 \mathrm{mg} / \mathrm{kg})$, and 3 a fourth dose $(10 \mathrm{mg} / \mathrm{kg})$. Neonates received different phenobarbital formulations: $10 \mathrm{mg} / \mathrm{mL}(n=20), 25 \mathrm{mg} /$ $\mathrm{mL}(n=11), 50 \mathrm{mg} / \mathrm{mL}(n=8)$, or combinations $(n=6)$.

Administration of $10 \mathrm{mg} / \mathrm{mL}$ phenobarbital resulted in higher cumulative PG dose $(1,400[431] \mathrm{mg} / \mathrm{kg})$ than 25 $\mathrm{mg} / \mathrm{mL}(426[288] \mathrm{mg} / \mathrm{kg})$ and $50 \mathrm{mg} / \mathrm{mL}(210$ [140] mg/ $\mathrm{kg})(p<0.001)$. Different phenobarbital formulations had similar lactate on MRS.

With regard to MRS, $P G_{\text {observed }}$ was not correlated with its metabolite lactate (Spearman's rho 0.18, $p=0.2$ ). Neither ethanol nor pyruvate was detected on MRS, indicating their concentrations were below detection level, that is, well below approximately $0.1-0.2 \mathrm{mM}$ (considering high neonatal spectral quality). 

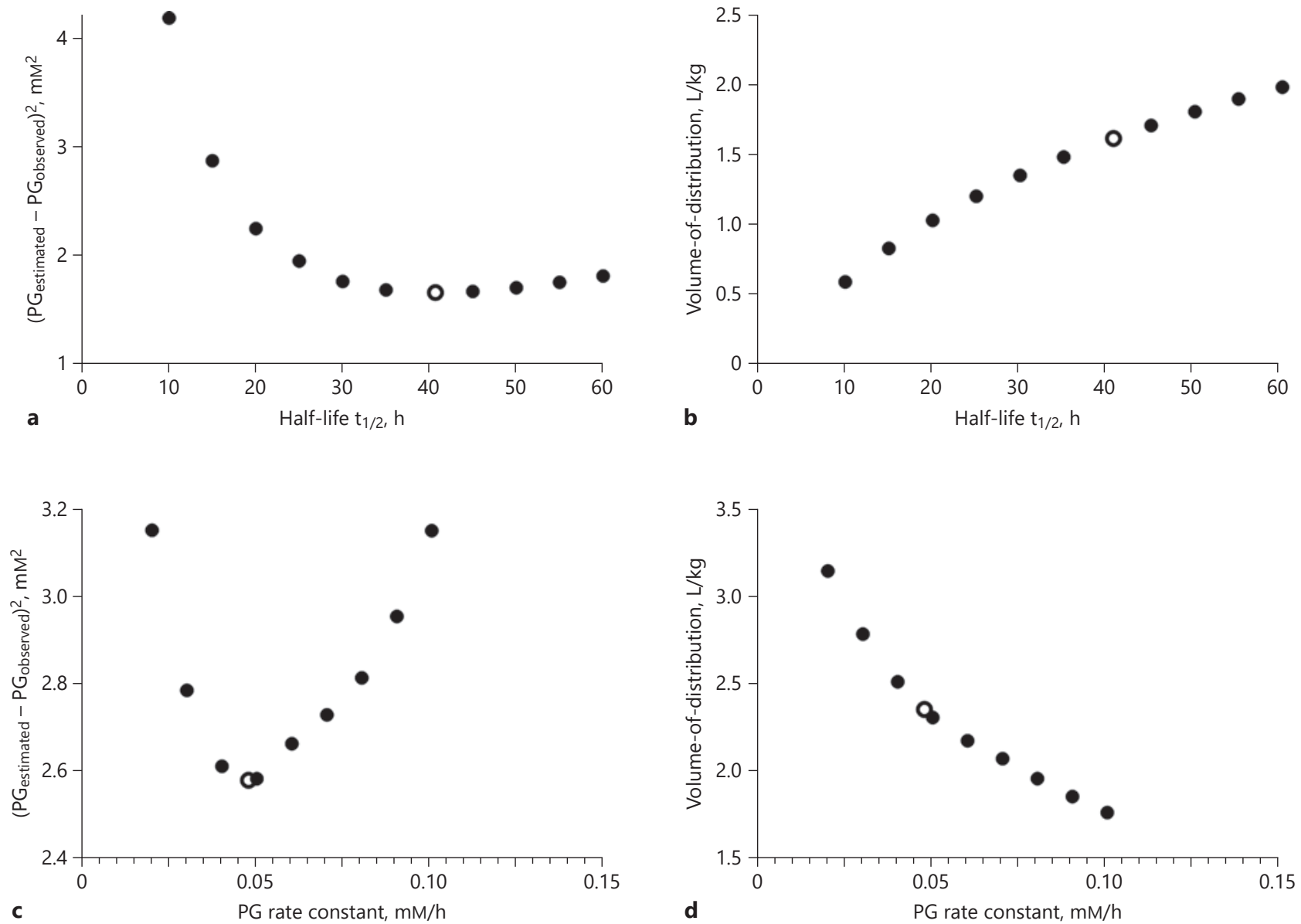

Fig. 3. Goodness of fit of PG clearance model. First-order model: squared difference between $P G_{\text {estimated }}$ and $P G_{\text {observed, }}$ that is, $\left(P G_{\text {estimated }}-P G_{\text {observed }}\right)^{2}$, versus half-life illustrating a broad minimum (a); corresponding volume of distribution versus PG half-life $\left(t_{1 / 2}\right)$ (b). Open dots illustrate a half-life of $40.7 \mathrm{~h}$ and a volume of distribution of $1.6 \mathrm{~L} / \mathrm{kg}$, leading to minimum ( $P G_{\text {estimated }}-$ $\left.P G_{\text {observed }}\right)^{2}$. Zero-order model: squared difference between

\section{Cerebral PG Clearance Model}

First-order kinetics resulted in a PG half-life of $40.7 \mathrm{~h}$ and volume of distribution of $1.6 \mathrm{~L} / \mathrm{kg}$ (Fig. 2a). Cumulative PG doses above or below the median were similarly distributed over the fit (Fig. 2b). The average squared difference between $P G_{\text {estimated }}$ and $P G_{\text {observed }}$ showed a flat minimum of $1.66 \mathrm{~mm}^{2}$ (Fig. 3a). When assuming lower half-life, the estimated volume of distribution becomes smaller (Fig. 3b) at the cost of an increased squared difference.

Zero-order kinetics showed a rate constant $k$ of 0.048 $\mathrm{mM} / \mathrm{h}$ and a volume of distribution of $2.35 \mathrm{~L} / \mathrm{kg}$ (Fig. $2 \mathrm{c}$ ). Cumulative PG doses above or below the median were
$P G_{\text {estimated }}$ and $P G_{\text {observed }}$, that is, $\left(P G_{\text {estimated }}-P G_{\text {observed }}\right)^{2}$, versus PG rate constant illustrating a steep minimum (c); corresponding volume of distribution versus PG rate constant (d). Open dots illustrate a PG rate constant of $0.048 \mathrm{~mm} / \mathrm{h}$ and a volume of distribution of $2.35 \mathrm{~L} / \mathrm{kg}$, leading to minimum $\left(P G_{\text {estimated }}-P G_{\text {observed }}\right)^{2}$. PG, propylene glycol.

similarly distributed over the fit (Fig. 2d). The minimum average squared difference between $P G_{\text {estimated }}$ and $P G_{\text {observed }}$ of $2.58 \mathrm{~mm}^{2}$ for this model was clearly higher than for first-order kinetics, making the first-order model superior (Fig. 3c, d).

\section{Clinical Diagnosis}

Four of the 22 asphyxiated neonates did not receive therapeutic hypothermia, due to prematurity $(n=1)$ or lack of encephalopathy $(n=3)$. Asphyxiated neonates with and without therapeutic hypothermia did not differ, except for higher postnatal age at MRS in those with hy- 
Table 2. Demographics and clinical data by diagnosis

\begin{tabular}{|c|c|c|c|c|c|}
\hline Mortality ${ }^{a}, n, \%$ & $8(36)$ & $1(25)$ & $2(20)$ & $1(25)$ & $1(20)$ \\
\hline Birth weight, $g$ & $3,168(772)$ & $3,350(1,077)$ & $3,102(994)$ & $3,545(1,439)$ & $2,854(1,099)$ \\
\hline \multicolumn{6}{|l|}{ Cumulative dose } \\
\hline Phenobarbital, mg/kg & $35(20)$ & $40(9.4)$ & $40(2.7)$ & $35.5(17)$ & $30(15)$ \\
\hline \multicolumn{6}{|l|}{ Cerebral MRI/MRS } \\
\hline Postnatal age at MRS, $\mathrm{h}$ & $113.5(26.3)^{1}$ & 105 (352.5) & $42(39.3)$ & $295(458.6)$ & $30(-)$ \\
\hline SNR & $16.4(4.3)$ & $14.3(3.6)$ & $15.8(4.5)$ & $13.5(3.9)$ & $17.4(4.4)$ \\
\hline $\mathrm{FWHM}, \mathrm{Hz}$ & $1.7(1.1)$ & $1.7(4.5)$ & $1.5(1.1)$ & $1.2(0.9)$ & $2.0(0.5)$ \\
\hline MRS PG, mM & $0.2(1.6)^{2}$ & $4.3(4.4)$ & $3.0(3.7)$ & $2.3(6.5)$ & $2.2(5.9)$ \\
\hline MRS lactate, mM & $0.85(1.1)$ & $0.9(1.5)$ & $0.7(1.2)$ & $1.2(0.8)$ & $0.6(0.3)$ \\
\hline
\end{tabular}

Between-group comparisons by the Kruskal-Wallis test and Bonferroni correction for multiple testing. Parameters are reported as median (IQR), except for mortality as frequency (\%) and SNR as mean \pm SD. FWHM, full-width at half-maximum; MRS, MR spectroscopy; PG, propylene glycol; SNR, signal-to-noise ratio; SD, standard deviation. ${ }^{a}$ Median postnatal age at death: asphyxia $188 \mathrm{~h}$ and stroke $90 \mathrm{~h}$. ${ }^{\mathrm{b}} \triangle \mathrm{PG}=P G_{\text {predicted }}$ (i.e., by $\mathrm{PG}$ clearance model) $-P G_{\text {observed }}$ (i.e., measured with MRS). ${ }^{1}$ Perinatal asphyxia versus perinatal stroke, $p<0.006$. ${ }^{2}$ Perinatal asphyxia versus perinatal stroke, $p=0.02$.

Fig. 4. $\triangle \mathrm{PG}$ by diagnosis. $\triangle \mathrm{PG}$, cerebral $P G_{\text {predicted }}$ (i.e., by $P G$ first-order clearance model) - cerebral $P G_{\text {observed }}$ (i.e., measured with MRS). No significant differences between groups. PG, propylene glycol; MRS, MR spectroscopy.

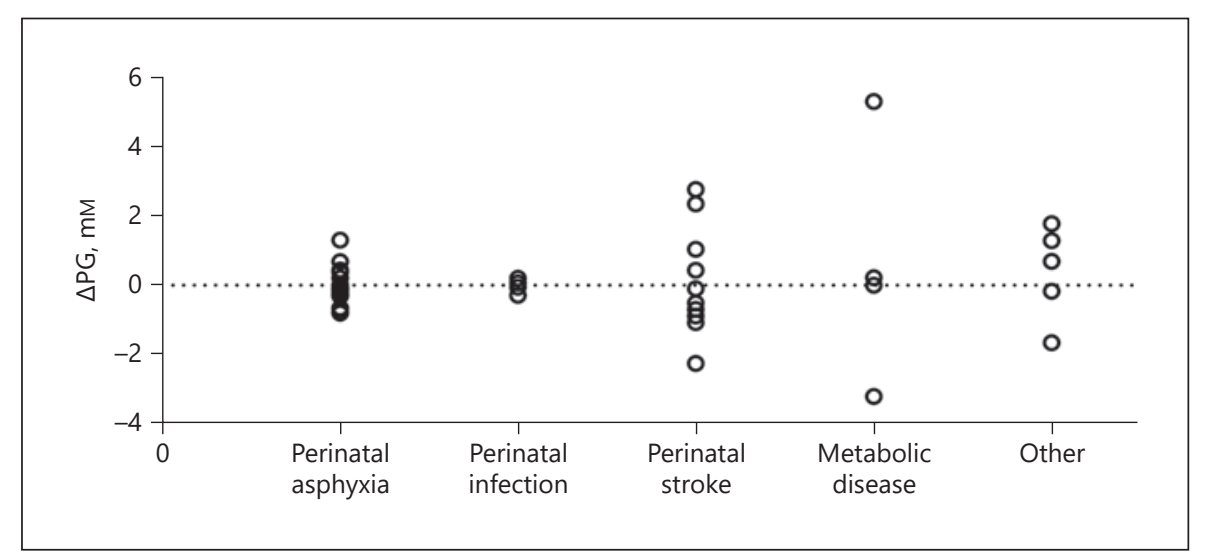

pothermia as MRI/MRS was performed after normothermia restoration (online suppl. Table). In other groups, some neonates fulfilled perinatal asphyxia criteria, that is, Apgar score $\leq 5$ or biochemical criteria (infection $n=2$; stroke $n=2$; metabolic disease $n=1$; other $n=1$ ); none were eligible for therapeutic hypothermia.

Population characteristics, spectral quality parameters, and MRS lactate were not different between diagnoses (Table 2). $P G_{\text {observed }}$ was lower in neonates with perinatal asphyxia versus stroke. Due to hypothermia-related delay, neonates with perinatal asphyxia had higher postnatal age at MRS particularly than stroke (Table 2; online suppl. Figure).
With first-order kinetics, $\triangle \mathrm{PG}$ (i.e., $P G_{\text {predicted }}-$ $P G_{\text {observed }}$ ) was not different between groups (Table 2; Fig. 4). In asphyxiated neonates, $\Delta \mathrm{PG}$ was not different between those with and without therapeutic hypothermia (online suppl. Table).

\section{Discussion/Conclusion}

This study demonstrated a long PG half-life of $40.7 \mathrm{~h}$ in neonatal brains. Assuming fast BBB diffusion, plasma half-life may also be longer than previously reported, 10.8-30.5 h [3, 6]. Diminished hepatic and renal clear- 
ance result in long neonatal plasma PG half-life compared to children and adults [3-5]. In our study, phenobarbital administration caused cerebral PG. Neonates received a median cumulative PG dose of $700 \mathrm{mg} / \mathrm{kg}$ [10], far exceeding the neonatal safety threshold of $1 \mathrm{mg} / \mathrm{kg} /$ day [8]. Combined with long cerebral PG half-life, neonates may have short-term $[6,8,11]$ and possible longterm adverse effects, which, to date, are not clearly defined in humans. High/repeated PG exposure results in cerebral apoptosis in mice and may result in short-term brain disturbances in infants [20]. However, it remains unknown if this results in long-term cognitive/behavioural abnormalities [20].

Neonatal brain PG concentrations might relate to changed $\mathrm{BBB}$ permeability due to perinatal asphyxia [12, 14], infection [13], or stroke [15], influencing passive BBB passage of PG. Hypoxia-ischemia causes endothelial injury, systemic inflammation, and reperfusion, increasing BBB permeability $[12,14,15]$. We showed similar PG clearance in asphyxia with or without therapeutic hypothermia, although differences cannot be excluded as groups were small. However, hypothermia does not affect phenobarbital clearance, after accounting for weight and postconceptional age $[21,22]$. More data are needed to evaluate if hypothermia influences cerebral PG clearance. Cerebral infection upregulates transcription factors that downregulate and remodel tight junctions, resulting in increased BBB permeability [13]. We demonstrated similar $\triangle \mathrm{PG}$ in neonates with perinatal asphyxia, infection, stroke, and metabolic disease, although we noticed large inter-subject variation in neonates with stroke and metabolic disease and the subgroups were small. Based on these indirect findings, we speculate that PG brain penetrance and clearance might not be related to BBB disruption. However, we cannot exclude certain conditions resulting in cerebral PG retainment. In addition, we assumed that $P G_{\text {observed }}$ is representative for actual cerebral concentrations. Although PG detection by $1 \mathrm{H}-\mathrm{MRS}$ is correlated with CSF-PG [23], no gold standard is available. We cannot relate $P G_{\text {observed }}$ with plasma concentrations as these were unavailable.

Hepatic PG degradation results in lactate and pyruvate $[1,2]$. In our study, MRS lactate did not correlate with $P G_{\text {observed }}$ and lactate was not different between groups (Table 2). These findings may, among others, be influenced by postnatal age at MRS. Also, we hypothesize that other reasons of lactate production are possible, for example, tissue hypoxia, inflammation, or metabolic. Moreover, PG degradation to lactate is hepatic and not cerebral; high serum lactate may not result in cerebral lactate.
We did not detect pyruvate on MRS. Also, ethanol, another solvent of phenobarbital, was not detected. Ethanol is eliminated before PG via the same alcohol dehydrogenase pathway (Fig. 1). Thus, ethanol might be missed because MRS was not obtained shortly after phenobarbital administration. Furthermore, we may assume that PG half-life may be shorter, in the absence of ethanol. However, co-administration of PG and ethanol is common.

This study was the first to describe a cerebral PG clearance model, similar to plasma [3]. All neonates followed a consistent MRI/MRS protocol and received similar standard care. MR spectra had high quality without between-group quality differences.

There were several limitations. First, we acknowledge limitations of retrospective studies. A prospective design is expected to be ethically challenging. High PG doses were unexpected and ascribed to market withdrawal of phenobarbital formulation. Formulation options with regard to phenobarbital/PG concentrations were limited. Still, this study is important for awareness of high cerebral concentrations and diminished clearance after PG-containing drugs. Second, the retrospective design limited sample size and data availability. Consequently, power analysis was unavailable. Also, diagnoses were unequally distributed with small groups, influencing statistical possibilities. Several criteria defined perinatal asphyxia. Overlap of these criteria between diagnostic groups may increase similarity and decrease differences. Third, the PG clearance model assumed population-based kinetics independent of gestational age, body weight, and metabolism. Because almost all neonates were term-born, gestational age differences were small. We concluded that first-order kinetics were more appropriate to describe our study population than zero-order kinetics. Nonetheless, the role of zero-order kinetics in high PG doses cannot be excluded. Unfortunately, we cannot model combined first- and zero-order kinetics as consistent intra-individual longitudinal data and short dose-to-MRI intervals are lacking. Also, we only focused on PG clearance after phenobarbital administration and did not take possible competition between ethanol and PG into account, both alcohol dehydrogenase substrates [24]. PG half-life might be shorter in the absence of ethanol as excipient-excipient interaction is lacking. Thus, the current study's PG clearance is only valid for neonatal phenobarbital containing PG and ethanol. Lastly, the cerebral PG clearance model was not independently validated. Also, $P G_{\text {observed }}$ was not compared to serum-PG or CSF-PG as these were unavailable. Taken this into account, results need to be interpreted cautiously. 
In conclusion, this MRS study showed a long neonatal cerebral PG half-life of $40.7 \mathrm{~h}$ based on first-order kinetics. Furthermore, PG clearance was similar in neonates with different diagnoses, but small groups should be accounted for. This might increase awareness of possible toxic neonatal cerebral PG concentrations due to intravenous PG-containing drugs. High PG administration should be avoided. MRS should be strongly considered when performing neonatal MRI with analysis including PG and ethanol in case of PG-containing drugs.

Future studies should compare neonatal brain and plasma PG, ethanol and lactate in asphyxia, stroke, and infection to make firmer statements about BBB changes possibly influencing cerebral PG exposure and toxicity. Furthermore, the clearance model we presented should be validated in neonatal controls. Also, intra-individual longitudinal data with different, including short, PGdose-to-MRS intervals may elucidate the presence of zero-order kinetics after high neonatal PG dosages.

\section{Statement of Ethics}

As evaluated by the Medical Ethics Review Committee of Amsterdam UMC, Amsterdam, The Netherlands, the Medical Research Involving Human Subjects Act did not apply to this study; therefore, there was waiver of informed consent. Furthermore, the study is exempt from ethical committee approval.

\section{Conflict of Interest Statement}

The authors have no conflicts of interest to declare.

\section{Funding Sources}

No funding was obtained for this study.

\section{Author Contributions}

Dr. van de Lagemaat and Dr. Pouwels conceptualized and designed the study, collected data, carried out the initial analyses, drafted the initial manuscript, and reviewed and revised the manuscript. Dr. van de Pol, Dr. Zonnenberg, and Dr. Witjes conceptualized and designed the study and critically reviewed the manuscript for important intellectual content. All authors approved the final manuscript as submitted and agreed to be accountable for all aspects of the work.

\section{Data Availability Statement}

De-identified individual participant data will not be made available. Group data may be provided upon specific request.

\section{References}

1 Speth PA, Vree TB, Neilen NF, de Mulder PH, Newell DR, Gore ME, et al. Propylene glycol pharmacokinetics and effects after intravenous infusion in humans. Ther Drug Monit. 1987 Sep;9(3):255-8.

2 Wilson KC, Reardon C, Theodore AC, Farber HW. Propylene glycol toxicity: a severe iatrogenic illness in ICU patients receiving IV benzodiazepines: a case series and prospective, observational pilot study. Chest. 2005 Sep; 128(3):1674-81.

3 De Cock RF, Knibbe CA, Kulo A, de Hoon J, Verbesselt R, Danhof M, et al. Developmental pharmacokinetics of propylene glycol in preterm and term neonates. Br J Clin Pharmacol. 2013 Jan;75(1):162-71.

4 Shehab N, Lewis CL, Streetman DD, Donn SM. Exposure to the pharmaceutical excipients benzyl alcohol and propylene glycol among critically ill neonates. Pediatr Crit Care Med. 2009 Mar;10(2):256-9.

5 Whittaker A, Currie AE, Turner MA, Field DJ, Mulla H, Pandya HC. Toxic additives in medication for preterm infants. Arch Dis Child Fetal Neonatal Ed. 2009 Jul;94(4): F236-40.
6 Glasgow AM, Boeckx RL, Miller MK, MacDonald MG, August GP, Goodman SI. Hyperosmolality in small infants due to propylene glycol. Pediatrics. 1983 Sep;72(3):353-5.

7 Seventeenth Report of Joint Food and Agriculture Organization/WHO Expert Committee on Food additives. Toxicological of certain food additives with a review of general principles and of specifications, technical report series No. 539. Geneva; 1974.

8 European Medicine Agency. Questions and answers on propylene glycol used as an excipient in medicinal products for human use. Committee for Human Medicinal Products; 2017.

9 Allegaert K, Vanhaesebrouck S, Kulo A, Cosaert K, Verbesselt R, Debeer A, et al. Prospective assessment of short-term propylene glycol tolerance in neonates. Arch Dis Child. 2010 Dec;95(12):1054-8.

10 Pouwels PJW, van de Lagemaat M, van de Pol LA, Witjes BCM, Zonnenberg IA. Spectroscopic detection of brain propylene glycol in neonates: effects of different pharmaceutical formulations of phenobarbital. J Magn Reson Imaging. 2019 Apr;49(4):1062-8.
11 Lim TY, Poole RL, Pageler NM. Propylene glycol toxicity in children. J Pediatr Pharmacol Ther. 2014 Oct; 19(4):277-82.

12 Ek CJ, D’Angelo B, Baburamani AA, Lehner C, Leverin AL, Smith PL, et al. Brain barrier properties and cerebral blood flow in neonatal mice exposed to cerebral hypoxia-ischemia. J Cereb Blood Flow Metab. 2015 May;35(5):818-27.

13 Kim BJ, Hancock BM, Bermudez A, Del Cid N, Reyes E, van Sorge NM, et al. Bacterial induction of Snaill contributes to blood-brain barrier disruption. J Clin Invest. 2015 Jun;125(6):2473-83.

14 Durukan A, Marinkovic I, Strbian D, Pitkonen M, Pedrono E, Soinne L, et al. Postischemic blood-brain barrier leakage in rats: 1-week follow-up by MRI. Brain Res. 2009 Jul 14;1280:158-65.

15 Kratzer I, Chip S, Vexler ZS. Barrier mechanisms in neonatal stroke. Front Neurosci. 2014;8:359.

16 Wynn JL. Defining neonatal sepsis. Curr Opin Pediatr. 2016 Apr;28(2):135-40.

17 Provencher SW. Estimation of metabolite concentrations from localized in vivo proton NMR spectra. Magn Reson Med. 1993 Dec; 30(6):672-9. 
18 Oakden WK, Noseworthy MD. Propylene glycol is essential in the LCModel basis set for pediatric $1 \mathrm{H}-\mathrm{MRS}$. J Comput Assist Tomogr. 2005 Jan;29(1):136-9.

19 Kulo A, de Hoon JN, Allegaert K. The propylene glycol research project to illustrate the feasibility and difficulties to study toxicokinetics in neonates. Int J Pharm. 2012 Oct 5; 435(2):112-4.

20 Lau K, Swiney BS, Reeves N, Noguchi KK, Farber NB. Propylene glycol produces excessive apoptosis in the developing mouse brain, alone and in combination with phenobarbital. Pediatr Res. 2012 Jan;71(1):54-62.

21 Shellhaas RA, Ng CM, Dillon CH, Barks JD, Bhatt-Mehta V. Population pharmacokinetics of phenobarbital in infants with neonatal encephalopathy treated with therapeutic hypothermia. Pediatr Crit Care Med. 2013 Feb; 14(2):194-202.

22 van den Broek MP, Groenendaal F, Toet MC, van Straaten HL, van Hasselt JG, Huitema $\mathrm{AD}$, et al. Pharmacokinetics and clinical efficacy of phenobarbital in asphyxiated new- borns treated with hypothermia: a thermopharmacological approach. Clin Pharmacokinet. 2012 Oct 1;51(10):671-9.

23 Cady EB, Lorek A, Penrice J, Reynolds EO, Iles RA, Burns SP, et al. Detection of propan-1,2-diol in neonatal brain by in vivo proton magnetic resonance spectroscopy. Magn Reson Med. 1994 Dec;32(6):764-7.

24 Valeur KS, Holst H, Allegaert K. Excipients in neonatal medicinal products: never prescribed, commonly administered. Pharmaceut Med. 2018;32(4):251-8. 